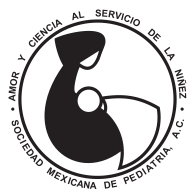

\title{
Efecto de una intervención educativa a padres de niños asmáticos en la adherencia al tratamiento
}

\author{
Carlos Coronel-Carvajal ${ }^{\ddagger, *}$ \\ ‡Hospital General Universitario “Armando E Cardoso”, Camagüey, Cuba.
}

\begin{abstract}
RESUMEN
Objetivo: Evaluar el impacto de una intervención educativa en padres de niños asmáticos sobre el conocimiento de la enfermedad y la adherencia al tratamiento. Material y métodos: Se realizó un estudio cuasiexperimental, en un hospital universitario en Cuba, de 2017 a 2019. Se incluyeron 202 padres de pacientes asmáticos menores de 19 años. Mediante cuestionarios se realizó evaluación del conocimiento y de la adherencia terapéutica, antes y después de un programa educativo. La interevención consistió en cinco encuentros, de una hora de duración, en los cuales se proporcionó información sobre el asma, los medicamentos (vía de administración y duración del tratamiento), así como de las consecuencias del incumplimiento del tratamiento. Resultados: De forma global, la intervención resultó benéfica: antes de la intervención $34.1 \%$ de los padres mostraron conocimientos suficientes sobre la enfermedad, incrementando a $85.6 \%$ después de la intervención. Mientras que la frecuencia de mala adherencia al tratamiento disminuyó tras la intervención, pasando de $73.7 \%$ a $11.4 \%$. Conclusiones: La intervención educativa mejoró el nivel de conocimiento de los padres sobre el asma, así como la adherencia al tratamiento.
\end{abstract}

Palabras clave: Asma, niños, cumplimiento terapéutico, intervención educativa.

\begin{abstract}
Objective: To assess the impact of an educational intervention on parents of asthmatic children, on the knowledge of the disease and in adherence to treatment. Material and methods: A quasi-experimental study was carried out in a university hospital in Cuba from 2017 to 2019; 202 parents of pediatric patients with asthma were included. Through questionnaires, knowledge and therapeutic adherence were evaluated before and after an educational program. The intervention consisted of five one-hour meetings, where information was provided regarding asthma, its medications (route of administration and duration of the treatment), as well as the consequences of non-compliance. Results: Overall, the intervention was beneficial: before the intervention $34.1 \%$ of the parents showed sufficient knowledge about the disease and, after the intervention, the percentage increased to $85.6 \%$. While, after the intervention, the frequency of poor adherence to treatment decreased, from $73.7 \%$ to $11.4 \%$. Conclusions: An educational intervention improved the parents' level of knowledge about asthma, as well as adherence to treatment.
\end{abstract}

Keywords: Asthma, children, therapeutic adherence, educational intervention.

\footnotetext{
*Correspondencia: CCC, camilo06@nauta.cu

Conflicto de intereses: El autor declara que no tiene.

Citar como: Coronel-Carvajal C. Efecto de una intervención educativa a padres de niños asmáticos en la adherencia al tratamiento. Rev Mex Pediatr. 2020; 87(2):51-57. doi: 10.35366/94168 [Effect of an educational intervention to parents of asthmatic children on treatment compliance]
}

\section{INTRODUCCIÓN}

El asma bronquial es una enfermedad inflamatoria crónica de las vías aéreas respiratorias que está presente en alrededor del 10\% de menores de 15 años, pero parece que su prevalencia ha aumentado en todo el mundo. Esta condición produce deterioro de la calidad de vida relacionada con salud, ya que se asocia con limitación de las actividades diarias, físicas y lúdicas. Además, interfiere con el sueño y el rendimiento académico, condiciona ausentismo escolar y ausentismo laboral en los padres. ${ }^{1}$ 


\begin{tabular}{|c|c|c|}
\hline \multirow[b]{2}{*}{ Conocimiento } & \multicolumn{2}{|c|}{ Suficiente } \\
\hline & $\begin{array}{l}\text { Antes } \\
\mathrm{n}(\%)\end{array}$ & $\begin{array}{c}\text { Después } \\
n(\%)\end{array}$ \\
\hline Conocimiento del asma & $65(32.2)$ & $192(95.0)$ \\
\hline Fármaco de elección & $34(16.8)$ & $188(93.1)$ \\
\hline Vía de administración & $49(24.2)$ & $186(92.1)$ \\
\hline Tiempo para evaluar la respuesta al tratamiento & $58(28.7)$ & $169(83.7)$ \\
\hline Duración del tratamiento & 47 (23.3) & $171(84.6)$ \\
\hline Consecuencias del incumplimiento del tratamiento & $19(9.4)$ & $177(87.6)$ \\
\hline
\end{tabular}

El objetivo del tratamiento del asma consiste en alcanzar y mantener el control clínico de la enfermedad, de acuerdo con las recomendaciones internacionales. En la mayoría de los pacientes, el control de la enfermedad se logra con educación, cambios ambientales de entorno del paciente, así como tratamiento a base de glucocorticoides inhalados. ${ }^{2}$ Sin embargo, aún persisten pacientes que no logran un control apropiado, lo cual puede relacionarse con la falta de cumplimiento de las indicaciones médicas, asociado al desconocimiento de la enfermedad y su tratamiento por sus padres. ${ }^{3}$ Cuando no hay cumplimiento terapéutico, no solamente los pacientes agravan su sintomatología, deterioran su capacidad funcional pulmonar y calidad de vida, sino que también aumentan el consumo de recursos sanitarios. ${ }^{4,5}$

Tanto el conocimiento de la enfermedad como la percepción de la misma son factores asociados al cumplimiento terapéutico. Este estudio tuvo como objetivo evaluar el impacto en la adherencia al tratamiento de una intervención educativa dirigida a padres de niños asmáticos.

\section{MATERIAL Y MÉTODOS}

Se realizó un estudio cuasiexperimental en el Hospital Universitario "Armando E Cardoso" de Guáimaro, provincia Camagüey, Cuba, en el periodo comprendido de mayo de 2017 a mayo de 2019.

De un total de 265 padres y/o representantes de niños menores de 19 años que acudieron a la consulta de pediatría con historia de asma, se consideraron elegibles 202 padres. Para su inclusión, los padres firmaron consentimiento informado, sus hijos eran > 5 años edad y con más de dos años del diagnóstico de asma, además de ser capaces de entender y completar los cuestionarios que se utilizaron para evaluar la intervención educativa.

El estudio se dividió en tres fases: la primera consistió en determinar el conocimiento básico de los padres sobre aspectos del asma, el tratamiento y el cumplimiento terapéutico. La segunda fue el programa educativo con cinco encuentros, de una hora de duración, en los cuales se proporcionó información sobre asma bronquial, los medicamentos de elección, vía de administración, duración y consecuencias del incumplimiento del tratamiento. En la tercera etapa se evaluó tanto el conocimiento adquirido como el cumplimiento terapéutico tres meses después de la intervención educativa.

Para determinar el nivel de conocimiento se aplicó el mismo cuestionario (Anexo 1) antes y después de la intervención educativa, el cual fue elaborado de conformidad con los contenidos de la intervención; antes de su aplicación fue validado por consenso. Como se observa, el cuestionario consta de seis preguntas, las cuales pueden tener de tres a cinco opciones de respuesta, para un total de 20 . Cada respuesta se contesta como verdadero (V) o falso (F). Cada opción correcta se le otorgó un valor de cinco puntos, de tal manera que el valor máximo es de 100 puntos.

La evaluación fue por pregunta y de manera global. Por pregunta, se consideró con conocimiento suficiente cuando se contestó correctamente las tres o cinco repuestas; por el contario, con una o más respuestas 
incorrectas, ese reactivo se contó como conocimiento insuficiente. Mientras que, de manera global, cuando el padre/madre alcanzó una puntuación de $\geq 70$ se determinó como conocimiento suficiente, de otra forma fue insuficiente.

Para evaluar la adherencia a la prescripción de los medicamentos se utilizó la escala Morisky-Green ${ }^{6}$, la cual fue modificada por el autor (Anexo 2). Son 10 preguntas que se contestan como "Si"o "No"; cuando se obtuvo $\geq 7$ se consideró buena adherencia terapéutica.

Análisis estadístico. Los datos se presentan con frecuencias simples y porcentajes. Para comparar los cambios en el conocimiento y la adherencia al tratamiento, antes y después de la intervención, se utilizó la prueba McNemar.

Los datos se procesaron utilizando el programa SPSS versión 25.0.

\section{RESULTADOS}

En la Tabla 1 se presentan las calificaciones de acuerdo con cada una de las seis preguntas, antes y después de la intervención educativa. Se observa que antes de la intervención alrededor del $30 \%$ de los padres tenían conocimiento suficiente de cada uno de los temas de las preguntas. Pero, después de la intervención, más del 80\% alcanzó conocimientos suficientes en las seis preguntas.

De forma global también se observó mejoría de los conocimientos. Antes de la intervención educativa sólo 69 padres de asmáticos (34.1\%) mostraron conocimientos suficientes sobre la enfermedad, y después de la intervención fueron 173 (85.6\%), lo cual fue estadísticamente significativo, $\mathrm{p}<0.001$ (Tabla 2).

En cuanto a la adherencia terapéutica, la intervención educativa mostró su beneficio, como se muestra en la Tabla
3. Previo a la intervención, 149 niños asmáticos (73.76\%) mostraban mala adherencia, lo cual cambió después de tres meses, ya que 181 pacientes (89.6\%) fueron evaluados con buena adherencia al tratamiento $(p<0.001)$.

\section{DISCUSIÓN}

El cumplimiento terapéutico se define como el grado en el que la conducta de un paciente coincide con la prescripción del médico en relación con la toma de los medicamentos, el seguimiento de una dieta o la modificación del estilo de vida. ${ }^{7,8}$ En este contexto, también se ha propuesto el término alianza terapéutica, para reflejar una relación más interactiva entre médico y paciente; identificando que la responsabilidad en seguir las instrucciones no sólo es del paciente, sino que también el médico debe conocer cómo y cuándo el paciente toma sus medicamentos. ${ }^{8}$

Las diversas formas de la deficiente adherencia al tratamiento incluyen: dificultades para el inicio del tratamiento, suspensión prematura o abandono, cumplimiento incompleto (omisión, error en dosis, tiempo), inasistencia a consultas. ${ }^{9-11}$

Se estima que una adherencia adecuada al tratamiento se logra cuando el medicamento es usado $80 \%$ o más de lo indicado. Sin embargo, se ha comprobado que la adherencia al tratamiento en pacientes asmáticos es baja, generalmente inferior a 50\%, lo cual aumenta su morbilidad. ${ }^{9}$

A juicio del autor, y de otros, uno de los principales factores relacionados con la adherencia terapéutica en niños asmáticos es la falta de conocimiento de los padres sobre la enfermedad, ${ }^{12,13}$ lo cual es trascedente, ya que son ellos quienes brindan o supervisan el cumplimiento a las indicaciones médicas. Por ejemplo, cuando

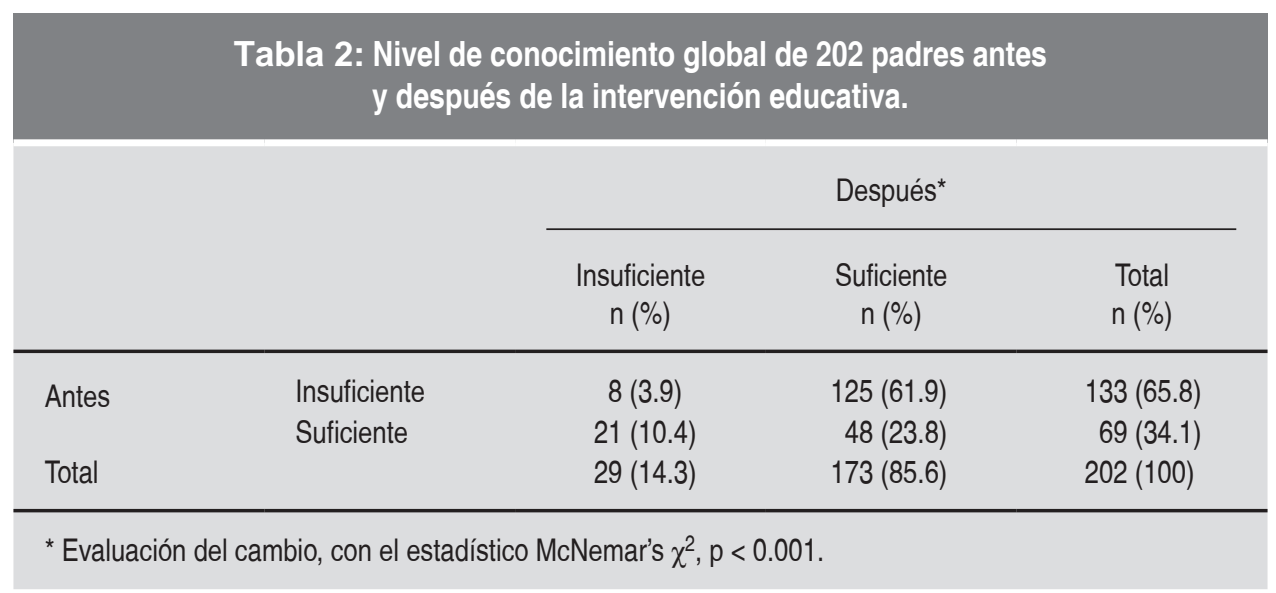




\begin{tabular}{|c|c|c|c|}
\hline \multirow[b]{2}{*}{ Antes } & \multicolumn{3}{|c|}{ Después* } \\
\hline & $\begin{array}{l}\text { Mala } \\
\mathrm{n}(\%)\end{array}$ & $\begin{array}{c}\text { Buena } \\
\mathrm{n}(\%)\end{array}$ & $\begin{array}{l}\text { Total } \\
\mathrm{n}(\%)\end{array}$ \\
\hline Mala & $11(5.4)$ & $138(68.3)$ & 149 (73.8) \\
\hline Buena & $10(4.9)$ & $43(21.3)$ & $53(26.2)$ \\
\hline Total & $21(10.4)$ & $181(89.6)$ & $202(100)$ \\
\hline
\end{tabular}

se desconoce que el asma es una enfermedad crónica e inflamatoria, no se podrá entender la importancia del tratamiento con esteroides inhalados, así cuando es necesario la combinación de tratamientos, o los efectos secundarios de los medicamentos. ${ }^{14}$ A partir de estas observaciones decidimos evaluar el posible efecto de una maniobra terapéutica y se pudo demostrar que, la mayor parte de los padres de niños asmáticos tenían pocos conocimientos básicos sobre el asma y su tratamiento, y además que sus hijos no cumplían con el tratamiento. Después de la intervención educativa, prácticamente todos los padres mejoraron su conocimiento, y como consecuencia, afortunadamente se incrementó la adherencia al tratamiento por sus hijos.

La falta de adherencia es un problema que conduce a mal control del asma, con aumento de las exacerbaciones y el riesgo de mortalidad. También se ha observado que otra consecuencia de la mala adherencia es la remodelación pulmonar, término que define una serie de cambios irreversibles a nivel pulmonar provocado por inflamación, y que puede aparecer tan precozmente, como a los tres años si el niño no recibe la terapia anti-inflamatoria. ${ }^{15}$ Además, cuando no hay apropiado cumplimiento, puede ocurrir que se valore de manera errónea el comportamiento del paciente asmático, poniendo en duda la efectividad de la terapéutica, ocasionando aumento innecesario de la dosis de los fármacos, con el consiguiente riesgo iatrogénico y la presentación de efectos secundarios, o bien, solicitar pruebas diagnósticas que no están justitificadas. ${ }^{16-18}$

\section{CONCLUSIONES}

Una intervención educativa dirigida a padres de niños asmáticos mejora el conocimiento de los padres y muy probablemente incrementa la adherencia al tratamiento de la enfermedad.

\section{REFERENCIAS}

1. Asensi Monzó MT, Duelo Marcos M, García Merino A. Manejo integral del asma en atención primaria. En: AEPap (ed.). Curso de Actualización Pediatría 2018. Madrid: Lúa Ediciones 3.0; 2018. Madrid: Lúa Ediciones 3.0; 2018. pp. 489-506.

2. Navarrete-Rodríguez E, Sienra-Monge JJL, Pozo-Beltrán CF. Asma en pediatría. Rev Fac Med UNAM. 2016; 59(4): 5-15.

3. Obando CD. Nivel de conocimiento de las madres sobre asma y adherencia al tratamiento en población pediátrica del consultorio externo de infecciones respiratorias del Hospital Nacional Hipólito Unanue, 2016 [Tesis para optar por el título profesional de Médico General]. Lima-Perú: Facultad de Medicina Humana. Universidad Ricardo Palma; 2017. Disponible en: http://cybertesis.urp.edu.pe/ bitstream/urp/926/1/DianaObando_2017_pdf.pdf.

4. Silva Germán E, Galeano E, Correa JO. Adherencia al tratamiento implicaciones de la no-adherencia. Acta Med Colomb. 2005; 30(4): 268-273.

5. Martín Alfonso L. Repercusiones para la salud pública de la adherencia terapéutica deficiente. Revista Cubana de Salud Pública. 2006; 32(3): [Aprox. 10 p.]. Disponible en: http://www. redalyc.org/articulo.oa?id=21420864013.

6. Morisky DE, Green LW, Levine DM. Concurrent and predictive validity of a self-reported measure of medication adherence. Medical Care. 1986; 24: 67-74.

7. López Romero LA, Romero Guevara SL, Parra DI, Rojas Sánchez LZ. Adherencia al tratamiento: Concepto y medición. Hacia Promoc Salud. 2016; 21(1): 117-137.

8. González GJ, Pérez EJ, García GL, Sánchez HJ, Criado-Álvarez JJ. Incumplimiento terapéutico. Rev Calid Asist. 2015; 30(1): 44-50.

9. Melero MC, Rodríguez MN, Díaz CR. Adherencia al tratamiento en asma. Situación actual. Rev Asma. 2017; 2(1): 13-22.

10. Kardas P, Lewek P, Matyjaszczyk M. Determinants of patient adherence: a review of systematic reviews. Front Pharmacol. 2013; 4: 91.

11. Villasis-Keever MA, Garduño-Espinosa J. Cumplimiento terapéutico en niños con cáncer. Bol Med Hosp Infan Mex. 1994; 51:706-712.

12. Coronel-Carvajal C. Influencia del conocimiento de los padres acerca del asma en el control del niño asmático. Rev Mex Pediatr. 2017;84(6):222-229. 
13. Bårnes CB, Ulrik CS. Asthma and adherence to inhaled corticosteroids: current status and future perspectives. Respir Care. 2015; 60: 455-468.

14. Adouni LM, Zongo F, Breton MC, Moisan J, Grégoire JP, Dorval $E$ et al. Factors associated with adherence to asthma treatment with inhaled corticosteroids: a cross-sectional exploratory study. J Asthma. 2018; 55(3): 318-329.

15. Huerta LJ, Jiménez GC, del Olmo TH, Maza LM. Remodelación de la vía aérea en asma. Alerg Asma Inmunol Pediatr. 2009; 18(2): 60-78.
16. Lindsay JT, Heaney LG. Non-adherence in difficult asthma and advances in detection. Expert Rev Respir Med. 2013; 76: 607614.

17. Pollard S, Bansback N, FitzGerald JM, Bryan S. The burden of nonadherence among adults with asthma: a role for shared decision-making. Allergy. 2017; 72: 705-712.

18. Blake KV. Improving adherence to asthma medications: current knowledge and future perspectives. Curr Opin Pulm Med. 2017; 23 (1): 62-70. 
Anexo 1: Cuestionario sobre conocimientos del asma.

1. El asma bronquial es una enfermedad:

Crónica _ $\mathrm{V}$

Aguda_F_

Inflamatoria _ $\mathrm{V}_{-}$

Controlable _ $\mathrm{V}_{-}$

Infecciosa_F_

2. El fármaco de elección en el control del asma enfermedad es:

Esteroides __

Broncodilatadores $-\mathrm{F}-$
Antihistamínicos $-\mathrm{V}_{-}$

3. La vía de elección para administrar los esteroides en el asma enfermedad es:

Inhalada__

Oral__ F_

Intravenosa _ $\mathrm{F}$

4. La respuesta al tratamiento con esteroides inhalados en el asma se evalúa:

a. Diariamente _ F

b. Mensualmente $-\bar{F}$

c. Cada tres meses $-\mathrm{V}_{-}$

5. La duración del tratamiento con esteroides inhalados en el asma:

Un mes_F_

Seis meses _ F

Hasta que el asma está controlada, con la mínima dosis posible de medicación durante al menos un año, y no hay riesgo de crisis _

6. El incumplimiento del tratamiento en el asma enfermedad causa:

Remodelación pulmonar _ $\mathrm{V}_{-}$

Mal control de la enfermedad _

Hospitalizaciones frecuentes _

\section{Evaluación:}

En la suma de las seis preguntas hay un total de 20 respuestas; por cada respuesta correcta se otorga cinco puntos, para un total de 100 puntos.

De manera global, $\geq \mathbf{7 0}$ puntos: conocimiento suficiente. Calificaciones inferiores: conocimiento insuficiente. 
Anexo 2: Escala Morisky-Green de adherencia a los medicamentos modificada ${ }^{6}$ para medir la adherencia al tratamiento para padres de niños con asma.

1. ¿Su hijo(a) cumple a diario el plan de tratamiento?

Sí (1) - No (0)

2. ¿Su hijo(a) hace uso de los corticoides inhalado diario?

Sí (1) - No (0)

3. ¿Usted está presente cuando usa el inhalado?

Sí (1) - No (0)

4. Cuando viaja, ¿olvida el medicamento?

Sí (0) - No (1)

5. ¿En la última semana olvidó el uso del medicamento?

Sí (1) - No (0)

6. Cuando observa que su hijo(a) lleva tiempo sin síntomas, ¿algunas veces deja de usar el medicamento?

Sí (0) - No (1)

7. ¿Sabe los beneficios que brinda el cumplimiento del tratamiento para su hijo(a) asmático(a)?

Sí (1) - No (0)

8. ¿Sabe por qué el médico le prescribió el medicamento a su hijo(a) asmático(a)?

Sí (1) - No (0)

9. ¿Considera que el plan de tratamiento indicado por el médico a su hijo(a) asmático es adecuado?

Sí (1) - No (0)

10. ¿Sabe usted cuánto dura el plan de tratamiento?

Sí (1) - No (0)

\section{Evaluación:}

$\geq 7$ : buena adherencia terapéutica

$\leq 6$ : mala adherencia terapéutica 